\title{
Equivalent circuit models for a biomembrane impedance sensor and analysis of electrochemical impedance spectra based on support vector regression
}

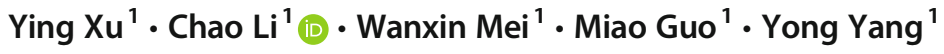

Received: 10 August 2018 / Accepted: 4 March 2019 / Published online: 2 April 2019

(C) The Author(s) 2019

\begin{abstract}
In this study, an electrochemical impedance biosensor was developed as a simple and fast method for real-time monitoring of biofilm binding properties via continuous impedance spectroscopy. To prepare the sensing membrane, cells were immobilized onto gold electrodes with nitrocellulose membranes. Different cell growth features were measured with the impedance instrument and analyzed using an equivalent model for data fitting and support vector regression (SVR) for data processing. The collected impedance spectra revealed that the binding attachment areas of cells differ depending on the cell density. Our results demonstrate the usefulness and feasibility of training our impedance-based sensor with a small amount of data to predict the effective area of different biofilms (GE, NGE, and CNGE), with a prediction error of $9.8 \%$.
\end{abstract}

Keywords Cell-nitrocellulose membrane $\cdot$ Electrochemical impedance spectra $\cdot$ Equivalent circuit models

\section{Introduction}

Electrochemical biosensors, often referred to as potentiometric, amperometric, impedimetric, or conductimetric sensors, are advantageous because they are highly sensitive, rapid, and inexpensive sensors that are also suitable for designing integrated microsystems [1]. The parameters obtained with electrochemical impedance spectroscopy (EIS) can represent different physical quantities [2-5]; thus, EIS can be used as a rapid, label-free, and sensitive process that meets the requirements of microscopic testing $[4,6]$. In electrochemical studies, qualitatively and even quantitatively measuring the physical quantities that correspond to the EIS parameters is important [6-8]. A new type of biological sensor was fabricated from an electrode, and an electrochemical biosensor membrane was formed by combining stationary substances with the corresponding detection substances to detect DNA and other biomolecules [9-11]. In addition, the relationship between the

Chao Li

503595003@qq.com

1 College of Life Information Science and Instrument Engineering, Hangzhou Dianzi University, No. 2 Road, Hangzhou, Zhejiang Province, China electrodes and the biofilm attachment can be used to study the active state of a biomembrane on an electrode surface $[10,12,13]$. Previous studies have shown that the fitted model of this relationship has good stability and prediction performance under different experimental conditions, and the model can predict the effective area of an electrode and provide a method for predicting the active surface area of a biomembrane electrode, which can reflect characteristics of the cell growth status, such as cell density [14-16].

A support vector machine (SVM) is a machine learning algorithm with a wide range of applications; it is especially good at building and forecasting two classification models. Support vector regression (SVR) is a regression method based on a SVM $[17,18]$. SVR has been widely used, always predicts data with high accuracy and can be used for quantitative analysis of physical and chemical quantities such as capacitance and resistance [19-21]. However, SVR has not been applied to the analysis of EIS for biosensors. In the present study, SVR is shown to have good accuracy and stability for predicting the active surface area on a biomembrane electrode.

The present work describes the use of EIS for equivalent circuit modeling and a comparison of electrochemical impedance data obtained with three different electrodes: a bare gold electrode (GE), a gold electrode with a nitrocellulose membrane (NGE), and a gold electrode with a cell-nitrocellulose membrane (CNGE). Based on the experimental data, we built 
an impedance model to obtain the required electrochemical parameters and fit the data with ZView (ZView Version 2.1c, Scribner Associates, Inc.). The main parameter was extracted as the eigenvector of the SVR by evaluating the impedance parameters using principal component analysis (PCA), which is a statistical procedure that uses an orthogonal transformation to convert a set of observations of possibly correlated variables into a set of linearly uncorrelated variables. Finally, the main parameter was used for data training and regression prediction.

\section{Methods}

\subsection{Materials}

$\mathrm{NaCl}, \mathrm{KCl}, \mathrm{Na}_{2} \mathrm{HPO}_{4} \cdot 12 \mathrm{H}_{2} \mathrm{O}, \mathrm{KH}_{2} \mathrm{PO}_{4}, \mathrm{~K}_{3}\left[\mathrm{Fe}(\mathrm{CN})_{6}\right]$, and $\mathrm{K}_{4}\left[\mathrm{Fe}(\mathrm{CN})_{6}\right] \cdot 3 \mathrm{H}_{2} \mathrm{O}$ were purchased from Sinopharm Chemical Reagent Co., Ltd.; nitrocellulose filter membranes were purchased from Biosharp Co., Ltd.; and human breast cancer cells (MCF-7) were purchased from Life Science Institute of Zhejiang University. All chemicals used in this work were analytical grade and used as received. All aqueous solutions were prepared using ultrapure water (ultrapure water produced by Aquelix 5).

\subsection{Preparatory experiments}

(1) Preparation of a $1 \mathrm{~mol} / \mathrm{L}$ PBS solution: $8 \mathrm{~g}$ of NaCl, $0.2 \mathrm{~g}$ of $\mathrm{KCl}, 3.63 \mathrm{~g}$ of $\mathrm{Na}_{2} \mathrm{HPO}_{4} \cdot 12 \mathrm{H}_{2} \mathrm{O}$, and $0.24 \mathrm{~g}$ of $\mathrm{KH}_{2} \mathrm{PO}_{4}$ were dissolved in $900 \mathrm{~mL}$ of deionized water, and the $\mathrm{pH}$ was adjusted to 7.4 using a hydrochloric acid solution. The solution was subsequently transferred to a 1-L volumetric flask and brought to its final volume with deionized water.

(2) Preparation of a $50 \mathrm{mM}$ buffer solution: $0.8231 \mathrm{~g}$ of $\mathrm{K}_{3}\left[\mathrm{Fe}(\mathrm{CN})_{6}\right]$ and $0.9200 \mathrm{~g}$ of $\mathrm{K}_{4}\left[\mathrm{Fe}(\mathrm{CN})_{6}\right] \cdot 3 \mathrm{H}_{2} \mathrm{O}$ were dissolved in a prepared PBS solution and brought to $100 \mathrm{~mL}$.

(3) Pre-treatment of electrodes: To ensure that the electrodes were flat and parallel, they were polished and then rinsed with deionized water. Subsequently, the electrodes were examined by cyclic voltammetry. The initial voltage was set to $-0.5 \mathrm{~V}$, the final voltage was set to $0.9 \mathrm{~V}$, and the scanning rate was set to $0.05 \mathrm{~V} / \mathrm{s}$. For subsequent operations, the difference in the redox peaks had to be less than $0.100 \mathrm{~V}$.

(4) Production of the cell-NC membrane: After a clean benchtop was sterilized for 15 min under continuous ultraviolet radiation, one test tube of MCF-7 cells (human breast cancer cell line, Life Science Institute of Zhejiang University) was taken from a liquid nitrogen box. Subsequently, the test tube was thawed in a $37^{\circ} \mathrm{C}$ thermostated water bath and then placed on the sterile benchtop. A $1 \mathrm{~mL}$ suspension was pipetted from the test tube into a centrifuge tube, and $4 \mathrm{~mL}$ of RPMI 1640 medium was added to the tube, which was subsequently placed into a low-speed centrifuge. The sample was centrifuged for $5 \mathrm{~min}$ at $800 \mathrm{r} / \mathrm{min}$, and its contents were then carefully mixed. Prior to the cells being transferred into a sterilized cell culture flask, the supernatant was discarded. Then, $4 \mathrm{~mL}$ of RPMI 1640 medium was added to the centrifuge tube and mixed. The flask was placed in a constant-temperature $\mathrm{CO}_{2}$ incubator, and the cells were cultured for $24 \mathrm{~h}$. Before the cell medium was exchanged, the cells were checked to ensure that they were in good condition. Afterwards, the cells were cultured for another $24 \mathrm{~h}$.

The NC membranes (NCEs) were cut into $3 \times 3 \mathrm{~cm}^{2}$ that were set aside after moist heat sterilization. Well-grown adherent cells were then selected, and the original culture solution was discarded. After digestion, a single-cell suspension was prepared from the original culture solution. Finally, $1 \mathrm{~mL}$ of the single-cell suspension was pipetted into a culture dish with the already prepared NC membranes, and RPMI 1640 was added to the culture dish. Afterwards, the culture dish was carefully placed in a $\mathrm{CO}_{2}$ incubator at $37{ }^{\circ} \mathrm{C}$ for 3 days (Fig. 1). For subsequent electrochemical experiments, an NC membrane with a modest growth density was selected (Fig. 1(b)).

\subsection{Electrochemical impedance spectroscopy}

The EIS experiment consisted of four main steps - an impedance test, data fitting, PCA and SVR - to gradually construct a biomembrane-electrode system.

Electrochemical measurements were performed using a $\mathrm{CH}$ Instruments $760 \mathrm{E}$ electrochemical analyzer $(\mathrm{CH}$ Instruments Inc., USA). One pole of the electrode was connected to the sensing probe on the electrochemical workstation, and the other pole was connected to both the reference and counter probes on the electrochemical workstation.

The tested frequency was set in the range from $1 \mathrm{~Hz}$ to $100 \mathrm{kHz}$ with a $50 \mathrm{mV}$ AC voltage in the impedance measurement program. Electrodes with cells at different densities were tested in wells containing $50 \mathrm{mM} \mathrm{K}_{4}\left[\mathrm{Fe}(\mathrm{CN})_{6}\right] / \mathrm{K}_{3}\left[\mathrm{Fe}(\mathrm{CN})_{6}\right]$ (1:1). For each condition ( 3 cell conditions $\times 5$ different electrodes), the EIS measurements were recorded 15 times, and the average value was used. After each measurement, the sensor was rinsed with PBS. All electrochemical measurements were performed at room temperature $\left(18-25{ }^{\circ} \mathrm{C}\right)$. To form a three-electrode system for impedance testing, we used a bare gold electrode, an NC film gold electrode, or a cell-NC film gold electrode as the working electrode, a platinum wire electrode as the counter electrode, and a silver/silver chloride 

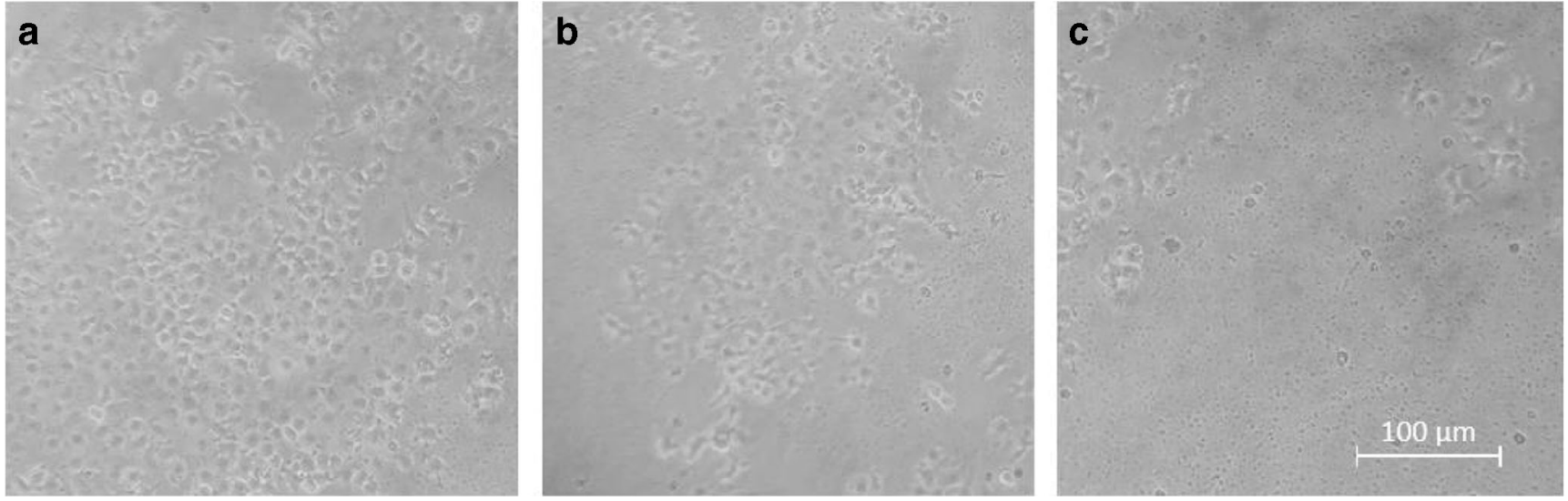

Fig. 1 MCF-7 cells of different densities (a) high growth density, (b) modest growth density, and (c) low cell density

electrode as the reference electrode. As shown in Fig. 2, the three experimental groups were recorded as GE, NGE, and CNGE, respectively.

The electrodes in each group were prepared with five different diameters of $1 \mathrm{~mm}, 2 \mathrm{~mm}, 3 \mathrm{~mm}, 4 \mathrm{~mm}$, and $5 \mathrm{~mm}$ for the impedance tests. In the NGE electrochemical impedance experiments, the $\mathrm{NC}$ film was attached to gold electrodes with different diameters to form an NC-membrane-electrode system for impedance testing. In the CNGE electrochemical experiment, NC membranes with different concentrations of MCF-7 cells were attached to gold electrodes with different diameters to form a cell-NC membrane electrode system for impedance testing.

An equivalent circuit model of the GE, NGE and CNGE groups was established based on the acquired EIS data. We then used the equivalent circuit to fit the data, analyzed the circuit components, and extracted the key parameters $\left(R_{s}\right.$, CPE-T, CPE-P, and $\mathrm{R}_{\mathrm{p}}$ ) by PCA as the input characteristics for SVR; the diameter served as the output for data training and prediction.

\section{Results}

\subsection{Equivalent circuit analysis}

From an electrical point of view, the performance of an electrochemical cell can be represented by an equivalent circuit that has the same behavior and output with an equivalent input

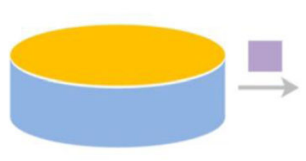

GE

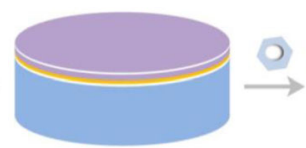

NGE

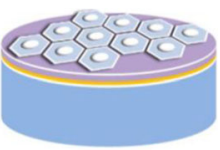

CNGE
Nitrocellulose membrane

(9) MCF-7 cell

Fig. 2 Schematic illustration of GE, NGE, and CNGE
[22-24]. To study the equivalent model of the biomembrane impedance sensor, the equivalent model of the impedance sensor without a biomembrane (GE) needed to be first analyzed $[25,26]$. According to the principles of electrochemical theory, the electrochemical reaction of a solution comprises two processes: the diffusion of ions from the solution to the interface of the electrode, i.e., the mass transfer process, and the ion reacting on the electrode, i.e., the activation process $[6$, $27,28]$.

To simplify a complex system, such as electrodes in contact with different electrolytes, the model of the impedance sensor can be considered equivalent to the classical Randles model [25, 29, 30] (Fig. 3). The circuit model comprises four elements: the ohmic resistance of the electrolyte solution $\left(\mathrm{R}_{\mathrm{S}}\right)$; the capacitance at the solution interface near the electrode, also known as the double-layer capacitance $\left(\mathrm{C}_{\mathrm{dl}}\right)$; the Warburg impedance $(\mathrm{W})$; and the charge transfer resistance $\left(\mathrm{R}_{\mathrm{p}}\right)$.

The Faraday impedance of the equivalent electrolyzer circuit for charge-transfer control and diffusion control includes two parts: $W$ and the electrode polarization resistance, $R_{p}$. Because the gold electrode used in this paper is an inert electrode, the Faraday impedance is very large. Thus, in this circuit, $\mathrm{R}_{\mathrm{s}}$ and $\mathrm{C}_{\mathrm{dl}}$ are approximated as ideal circuit elements, and the $\mathrm{R}_{\mathrm{p}}$ and $\mathrm{W}$ impedances are not ideal components; they have a certain relationship with the measuring frequency. Additionally, because the electrode surface was rough, we replaced the ideal capacitor element $\left(\mathrm{C}_{\mathrm{dl}}\right)$ with a constant

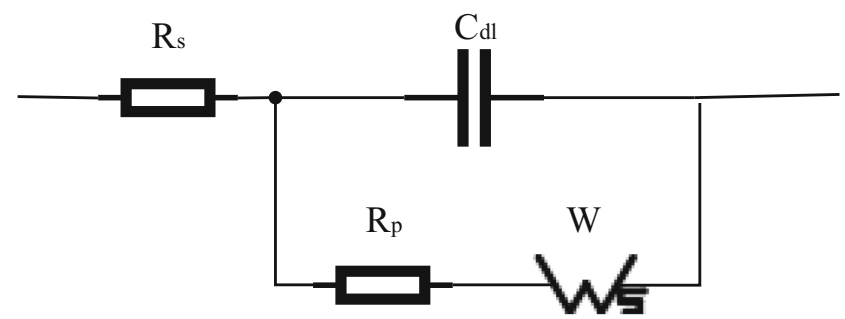

Fig. 3 The Randles equivalent circuit model 
phase element (CPE) [26, 29], which can improve the quality of the fitting (Fig. 4).

Compared with the GE, the NGE has an NC membrane that participates in ion exchange; thus, the equivalent circuits of the GE and NGE differ. The working electrode in this experiment was separated from the other two electrodes with an NC film, which is equivalent to a shielding layer on the working electrode; thus, the oxidized and reduced substances were prevented from exchanging electrons on the electrode [28, 30]. Normally, $R_{p}$ depends on the dielectric and insulating features at the electrode-electrolyte interface, but under these conditions, it was affected by the property changes occurring at the interface. Compared with GE, the immobilization of the nitrocellulose membrane changed the dielectric features at the interface, resulting in an increase in $\mathrm{R}_{\mathrm{p}}$. However, the capacitance between the metal and the membrane could be regarded as a CPE; thus, it was still equivalent to the CPE [31, 32]. In the equivalent circuit model of the NGE, the primary elements did not change; therefore, the NGE model was still equivalent to the equivalent model in Fig. 4.

The structure of the CNGE is similar to that of NGE; the difference between the CNGE and NGE is that MCF-7 cells are growing on the $\mathrm{NC}$ membrane in the CNGE. The NC membrane with cells can be regarded as a biofilm structure due to the adherence of cells. Thus, the equivalent circuit model of the CNGE can also be equivalent to the model in Fig. 4, but the resistance and the capacitance have changed. When the system applied a voltage to the cell-NC + membrane electrode, the current flowed through the electrode-NC membrane-cell system to reach the electrolyte. Thus, the resistance of the electrode-NC membrane cell could be considered to be in series with the resistance of the electrolyte, which can be described as resistance $\left(\mathrm{R}_{\mathrm{p}}\right)$ [28]. The capacitance between the metal and the cell-NC membrane can also be regarded as a constant (CPE) [30, 32]. According to the electrical characteristics, $R_{p}$ will increase, and the change in the constant will be more rapid.

To obtain the values of the parameters in the equivalent model, the impedance data were fitted with the equivalent model in ZView (Fig. 5), as previously discussed. The comparison between the raw data and the fitting data demonstrates the practical application of this method.

As shown in Fig. 4, the equivalent model we used was composed of 4 elements, and 7 electrical parameters $\left(R_{s}\right.$, CPE-T, CPE-P, $R_{p}, W-T, W-P$, and W-R) were generated. If the 7 parameters were all taken as inputs, the EIS analysis

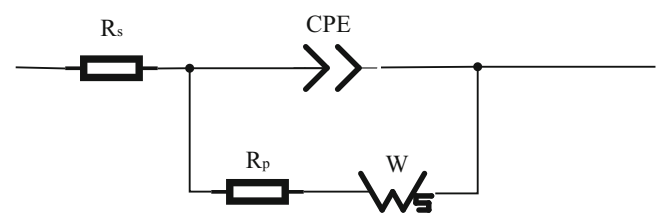

Fig. 4 Practical equivalent circuit model of the GE

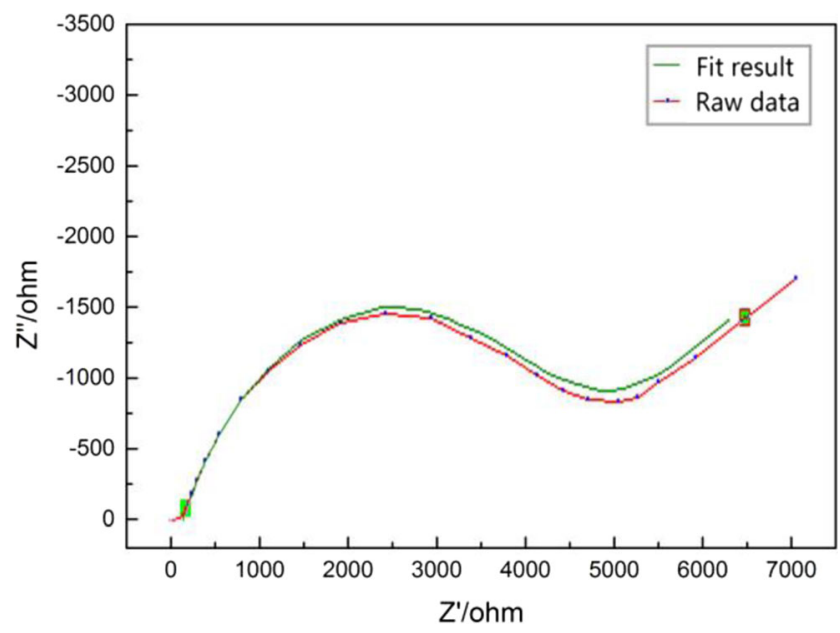

Fig. 5 Comparison between the raw data and the fitting data

would be a very complicated 7-dimensional problem. To simplify this complex problem, we first used PCA to address the 7 parameters, and then, we only analyzed the 4 parameters most related to the output. In this way, the 7-dimensional problem was simplified to a 4-dimensional problem, greatly reducing the workload while only slightly affecting the accuracy. The specific PCA is discussed in detail in Section 3.2.

\subsection{Analysis of the characteristic parameters}

The impedance spectra (Nyquist plots) of GEs with different diameters $(1 \mathrm{~mm}, 2 \mathrm{~mm}, 3 \mathrm{~mm}, 4 \mathrm{~mm}$, and $5 \mathrm{~mm})$ are shown in Fig. 6(a). With increasing electrode diameter, the impedance semicircle decreases in magnitude and diameter. The impedance spectra (Nyquist plots) of the GE, NGE, and CNGE are shown in Fig. 6(b). The impedance spectra are characterized by a semicircle portion at high frequencies, corresponding to the electron transfer-limiting process [6, 29]; the diameter of the semicircle is equal to the charge transfer resistance. When different substances are adsorbed onto an electrode surface, they disturb the charge-transfer process between the electrode and the electrolyte solution, resulting in changes in the charge transfer resistance. As shown in the figure, the diameter of the semicircle increased when the nitrocellulose membrane and MCF-7 cells were immobilized on the electrodes.

After fitting the Randles model, we obtained 7 parameters $\left(\mathrm{R}_{\mathrm{s}}, \mathrm{CPE}-\mathrm{T}, \mathrm{CPE}-\mathrm{P}, \mathrm{R}_{\mathrm{p}}\right.$, W-T, W-P, and W-R) [29, 30]. If all 7 parameters were used for regression prediction by SVR, the amount of data would be too large, and the operation speed would be too slow; such a large number of parameters would result in redundancy and a cumbersome analysis of the parameters. Therefore, PCA was used to analyze the correlation among the 7 parameters [31,32], and the most relevant parameters were considered the main components. The information for the 7 parameters, obtained by the princomp function in MATLAB, is shown in Table 1. 
a

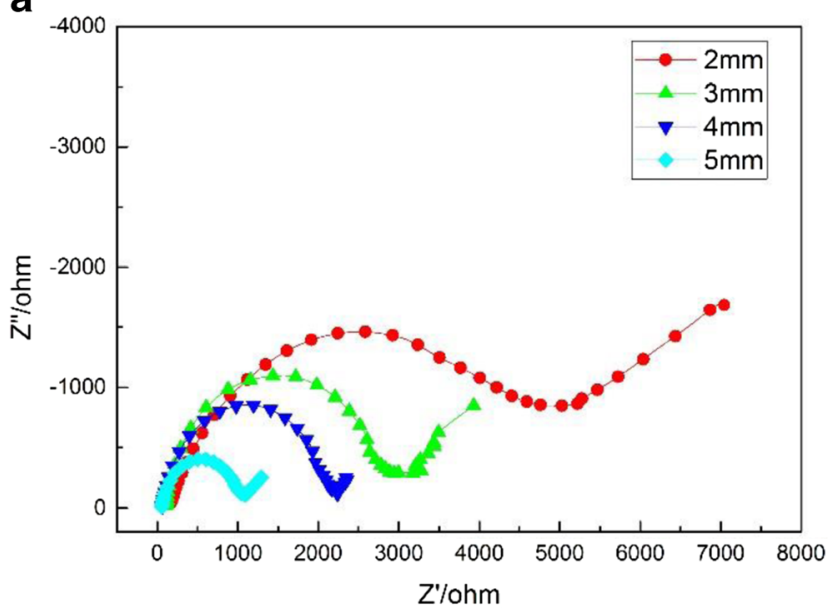

b

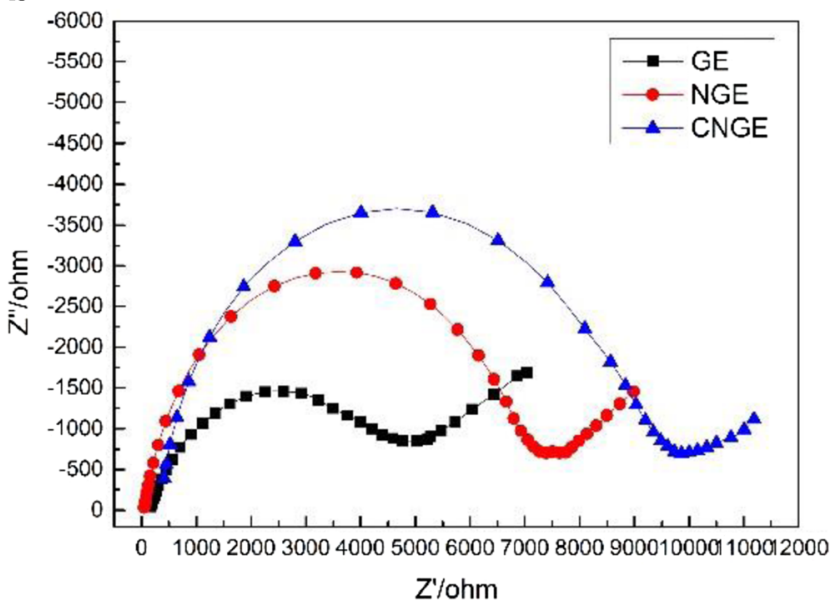

Fig. 6 (a) The impedance spectra of GEs with different diameters ( $2 \mathrm{~mm}$, $3 \mathrm{~mm}, 4 \mathrm{~mm}$, and $5 \mathrm{~mm}$ ) (b) The impedance spectra (Nyquist plots) of the GE, NGE, and CNGE with a gold electrode $(2 \mathrm{~mm})$

The four parameters with the highest relevance values of $48.75 \%, 19.03 \%, 13.71 \%$, and $10.50 \%$ were $\mathrm{R}_{\mathrm{s}}$, CPE-T, CPE-, and $R_{p}$, respectively. These results are consistent with the aforementioned equivalent circuit and verify the practical significance of the equivalent circuit.

The amount of information contained in the four parameters was $91.99 \%$, covering more than $80 \%$ of the circuit information. Thus, $R_{s}$, CPE-T, CPE-P, and $R_{p}$ can be selected as eigenvectors and used as input for SVR training and prediction.

\subsection{Analysis of data training and prediction}

$R_{s}$, CPE-T, CPE-P, and $R_{p}$ were used as inputs in the SVR model, and the electrode diameter was used as the output to

Table 1 Parameter information for the electrochemistry experiments

\begin{tabular}{llllllll}
\hline Parameter & $\mathrm{R}_{\mathrm{s}}$ & CPE-T & CPE-P & $\mathrm{R}_{\mathrm{p}}$ & $\mathrm{W}-\mathrm{T}$ & $\mathrm{W}-\mathrm{P}$ & $\mathrm{W}-\mathrm{R}$ \\
\hline Proportion (\%) & 48.75 & 19.03 & 13.71 & 10.50 & 5.52 & 2.01 & 0.39 \\
\hline
\end{tabular}

establish the SVR model of the electrode. For each of the three groups (GE, NGE, and CNGE), 100 data points were collected, corresponding to 20 data points for each diameter $(1 \mathrm{~mm}$, $2 \mathrm{~mm}, 3 \mathrm{~mm}, 4 \mathrm{~mm}$, and $5 \mathrm{~mm}$ ) in each group. A total of $35 \%$ of the data points were randomly selected as the training data (Fig. 7), with $15 \%$ used as the prediction data (Fig. 8). The results obtained from each test were compared with the actual results to determine the mean squared error (MSE), training error rate, and prediction error rate (Table 2). To ensure the best results in the training process, the best result was automatically adjusted by the program. Moreover, random extraction, training, and prediction of the training data ensured that the differences among the data obtained for each group had little effect on the overall results.

To randomly select 10 sets of data from each group, the three groups of impendence models were tested 50 times. On the basis of these selected data, 10 sets of data were tabulated. The three data sets contained the results for SVR implementation of impedance parameters for the GE, NGE, and CNGE. For the GE, the average MSE was $1.99 \mathrm{E}-03$, the average training error was $4.96 \%$, and the average prediction error was $4.90 \%$. For the NGE, the average MSE was $2.24 \mathrm{E}-03$, the average training error was $8.11 \%$, and the average prediction error was $11.20 \%$. For the CNGE, the average MSE was $1.29 \mathrm{E}-02$, the average training error was $12.19 \%$, and the average prediction error was $13.31 \%$. As shown in the table, the average MSE of the GE was $1.99 \mathrm{E}-03$, and its average training error and average prediction error were both less than $5 \%$; thus, we inferred that its accuracy reached $95 \%$. The average MSE values of the other two groups were approximately $2.24 \mathrm{E}-03$ and $1.29 \mathrm{E}-02$, and both the average training error and average prediction error were approximately $10 \%$, which demonstrate that these four electrochemical parameters are reasonable and effective for setting up SVR models. We also concluded that the fitted model has good stability and prediction performance under different experimental conditions. As such, it can predict the repetition rate difference of the effective area on the electrode array or alternatively provide a method for predicting the active surface area of electrodes with different shapes but the same area or the effective cell-attached active surface area in cell attachment experiments with different densities.

\section{Discussion}

In this paper, SVR was adopted to train and predict an evaluation model of a biomembrane electrode. The impedances with five effective electrode areas were tested under three different conditions (GE, NGE, and CNGE), and the electrochemical impedance spectra were fitted by modeling the corresponding equivalent circuits to determine the correlation between the effective electrode area and various 

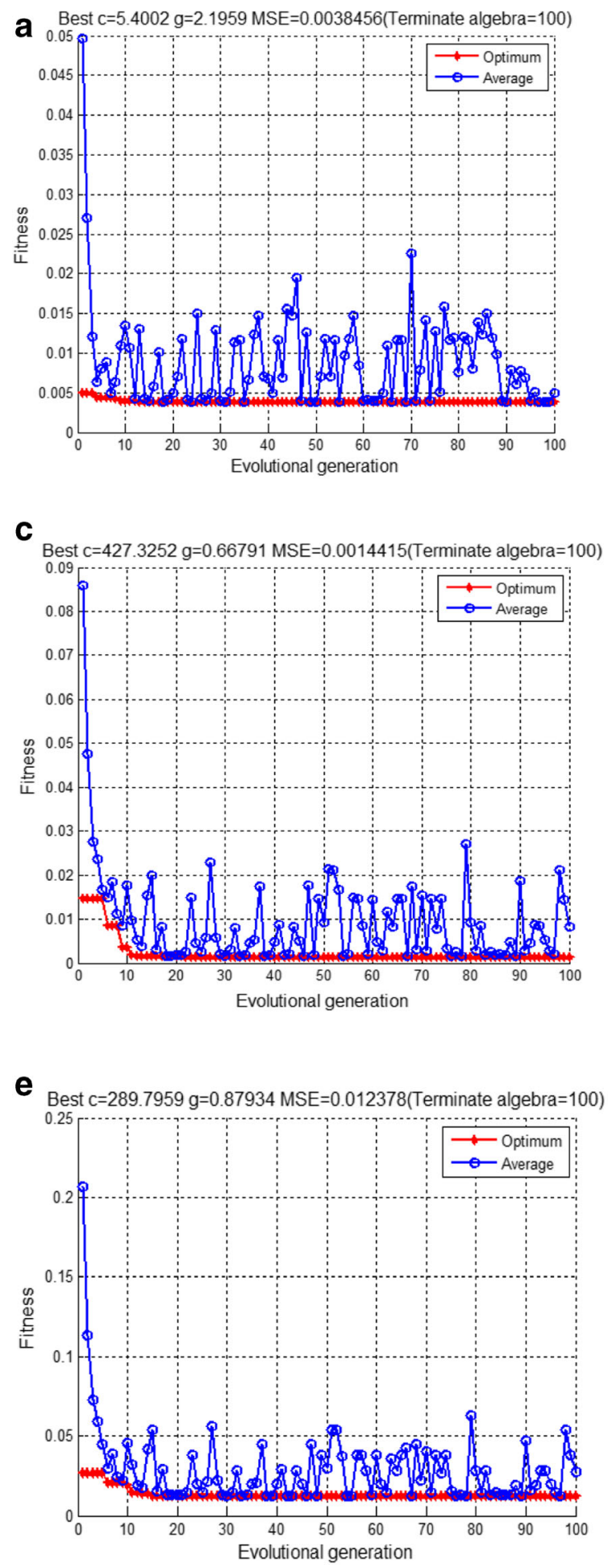
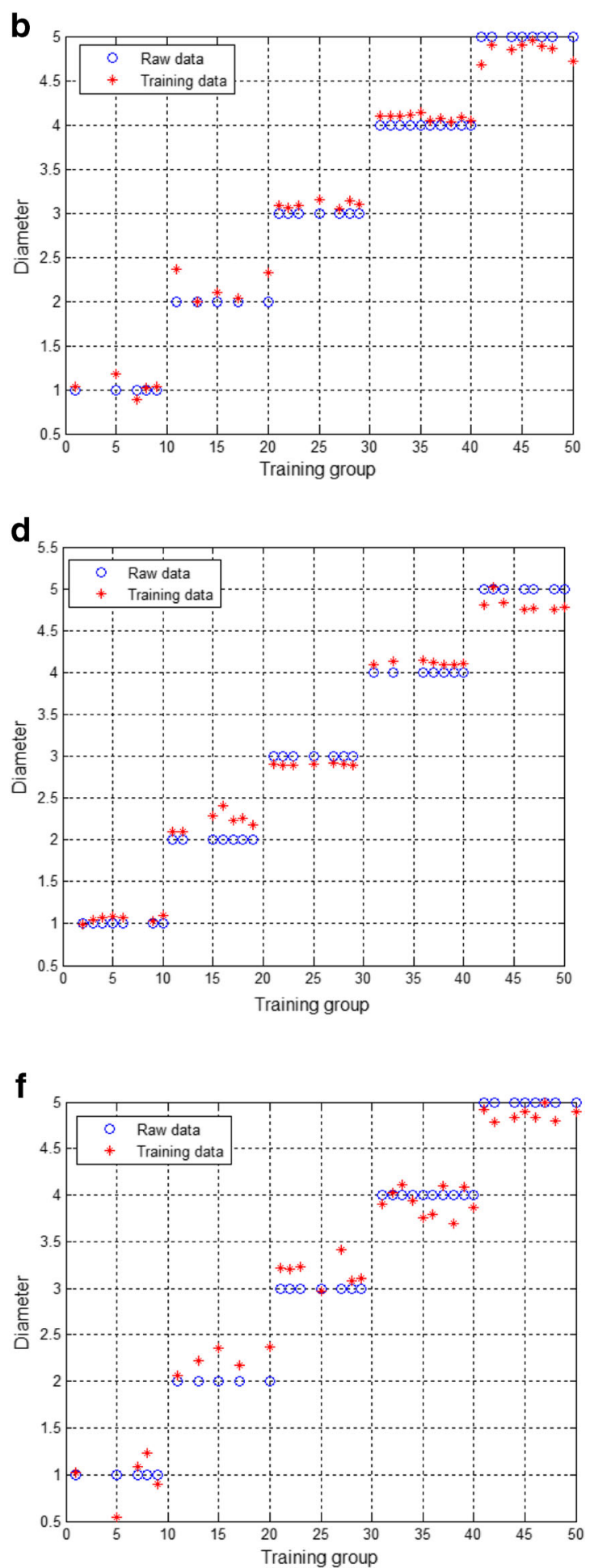

Fig. 7 (a) Training of the GE, (b) training results for the GE, (c) training of the NGE, (d) training results for the NGE, (e) training of the CNGE, and (f) training results for the $\mathrm{CNGE}$

electrochemical parameters. Based on PCA, key parameters $\left(\mathrm{R}_{\mathrm{s}}, \mathrm{CPE}-\mathrm{T}, \mathrm{CPE}-\mathrm{P}\right.$, and $\mathrm{R}_{\mathrm{p}}$ ) were extracted as the input characteristics for SVR, and the effective electrode area was used as the output to train and predict the data. The average error of the three groups was $9.8 \%$, that of the GE group was $4.9 \%$, and the average MSE was $5.71 \mathrm{E}-3$, which shows that the developed SVR model is accurate and widely applicable. We also expect that this model can be used to address both the problem of cell growth density on high-flux electrode arrays and the issue of determining the repetition rate of cell attachment on electrode arrays through a simple impedance test and SVR model. These problems are usually addressed 
a

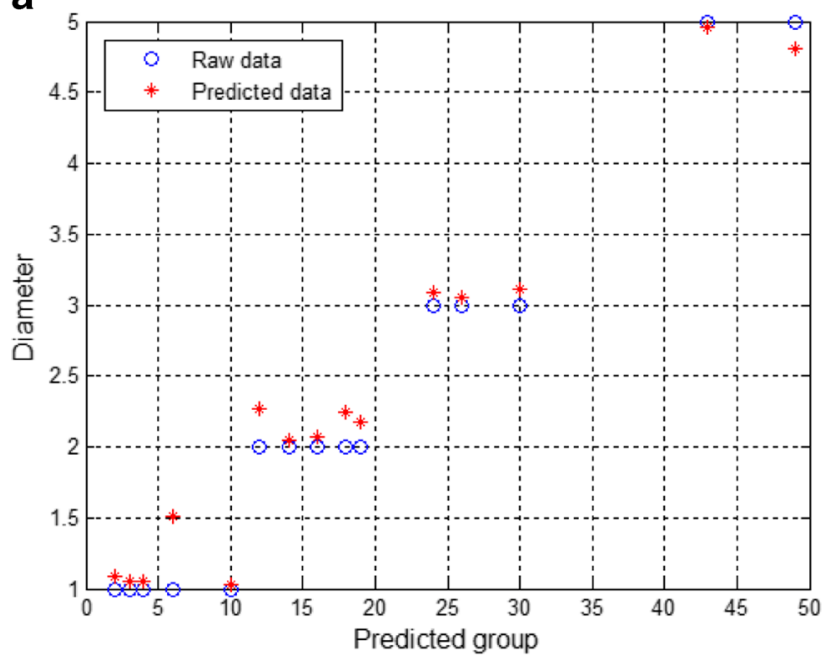

b

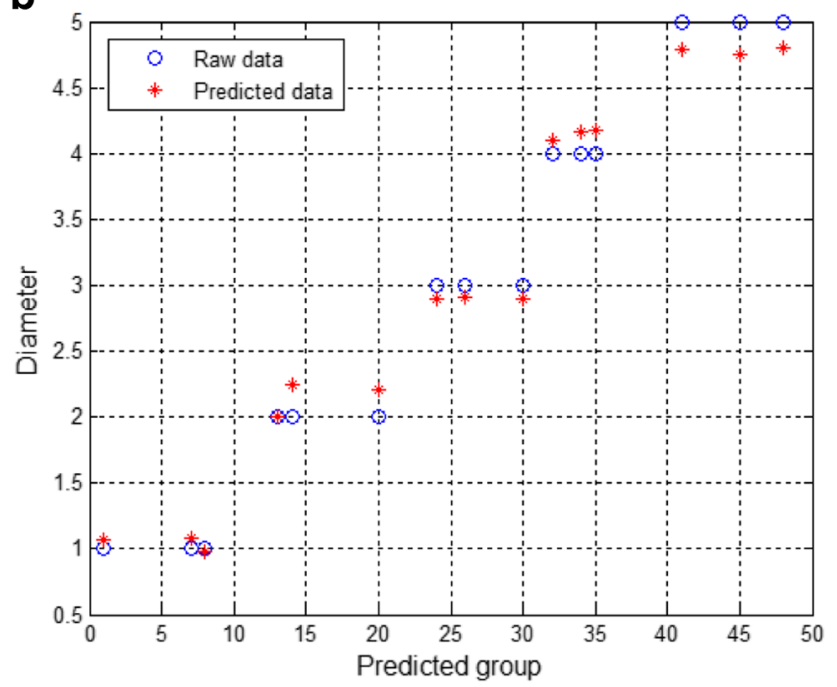

C

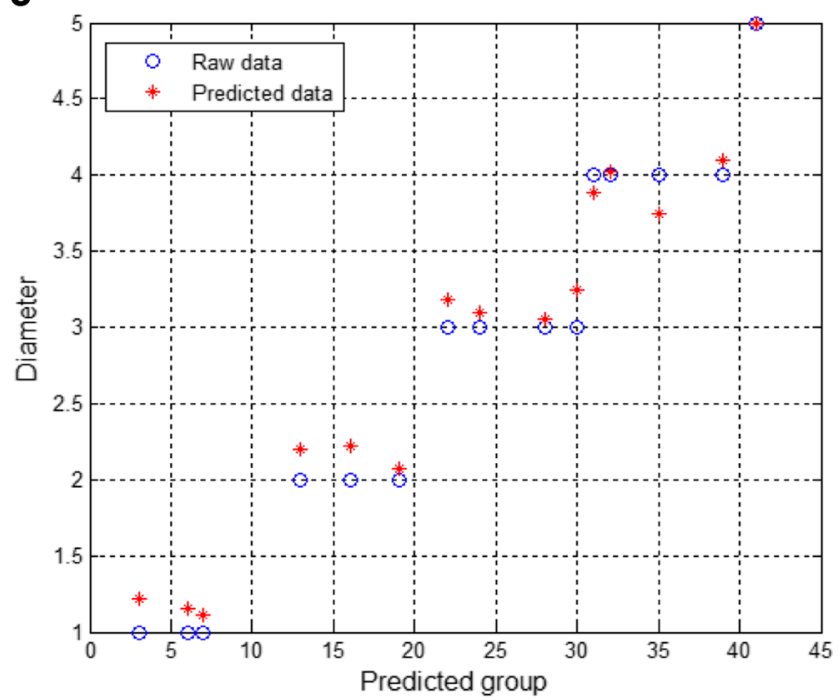

Fig. 8 (a) Prediction for the GE, (b) prediction for the NGE, (c) prediction for the CNGE
Table 2 Training results and analysis from 10 tests of the three electrical impedance models

\begin{tabular}{|c|c|c|c|}
\hline Model & MSE & Training error $\%$ & Prediction error $\%$ \\
\hline \multirow[t]{10}{*}{ GE } & $3.68 \mathrm{E}-04$ & 5.19 & 3.70 \\
\hline & $3.08 \mathrm{E}-04$ & 4.43 & 7.71 \\
\hline & $4.03 \mathrm{E}-03$ & 5.24 & 4.06 \\
\hline & $3.10 \mathrm{E}-03$ & 5.42 & 3.01 \\
\hline & $3.06 \mathrm{E}-03$ & 5.06 & 5.76 \\
\hline & $4.81 \mathrm{E}-03$ & 5.35 & 3.61 \\
\hline & $5.45 \mathrm{E}-04$ & 4.74 & 4.81 \\
\hline & $2.54 \mathrm{E}-03$ & 4.09 & 7.29 \\
\hline & $4.70 \mathrm{E}-04$ & 5.09 & 3.72 \\
\hline & $6.37 \mathrm{E}-04$ & 4.97 & 5.34 \\
\hline Average & $1.99 \mathrm{E}-03$ & 4.96 & 4.90 \\
\hline \multirow[t]{10}{*}{ NGE } & $7.92 \mathrm{E}-04$ & 8.60 & 12.27 \\
\hline & $9.26 \mathrm{E}-04$ & 6.36 & 9.44 \\
\hline & $1.05 \mathrm{E}-03$ & 8.68 & 11.57 \\
\hline & $5.34 \mathrm{E}-03$ & 7.55 & 7.67 \\
\hline & $4.07 \mathrm{E}-03$ & 7.94 & 14.60 \\
\hline & $3.44 \mathrm{E}-03$ & 7.48 & 16.81 \\
\hline & $9.32 \mathrm{E}-04$ & 8.89 & 12.64 \\
\hline & $2.91 \mathrm{E}-03$ & 8.89 & 8.02 \\
\hline & $7.11 \mathrm{E}-04$ & 8.57 & 7.82 \\
\hline & $7.92 \mathrm{E}-04$ & 8.60 & 12.27 \\
\hline Average & $2.24 \mathrm{E}-03$ & 8.11 & 11.20 \\
\hline \multirow[t]{10}{*}{ CNGE } & $6.12 \mathrm{E}-03$ & 10.25 & 13.55 \\
\hline & $1.18 \mathrm{E}-02$ & 13.66 & 7.82 \\
\hline & $2.01 \mathrm{E}-02$ & 13.01 & 13.39 \\
\hline & $1.76 \mathrm{E}-02$ & 12.94 & 14.01 \\
\hline & $2.16 \mathrm{E}-02$ & 12.61 & 13.99 \\
\hline & $9.48 \mathrm{E}-03$ & 12.53 & 16.44 \\
\hline & $7.58 \mathrm{E}-03$ & 10.78 & 14.13 \\
\hline & $1.37 \mathrm{E}-02$ & 13.17 & 12.01 \\
\hline & $7.57 \mathrm{E}-03$ & 11.17 & 16.88 \\
\hline & $1.35 \mathrm{E}-02$ & 11.80 & 10.85 \\
\hline Average & $1.29 \mathrm{E}-02$ & 12.19 & 13.31 \\
\hline
\end{tabular}

through microscopic observations. This model can also aid in the quantitative analysis and evaluation of the exact cell attachment rate of electrodes with different shapes but the same area (such as interdigital electrodes, disc electrodes, and diamond electrode arrays).

\section{Conclusion}

In conclusion, in this work, an electrochemical impedance biosensor based on SVR was developed to detect the effective area of a biomembrane. Under three different conditions (GE, NGE, and CNGE), the average prediction error of our 
biosensor was $4.9 \%$. This result indicates that the biosensor in this paper can provide a sensing platform to selectively and quantitatively detect the effective areas of different types of biomembranes. These results might help further work on the rapid detection of cell growth status and could even be used for detection and analysis in multidirectional microorganismrelated fields.

Funding information This work was supported by the National Natural Science Foundation of China (grant number 61871165) and the Public Welfare Project of the Zhejiang Provincial Science and Technology Department (grant number 2017C33055).

\section{Glossary}

CNGE Gold electrode with a cell-nitrocellulose membrane

EIS Electrochemical impedance spectroscopy

GE Bare gold electrode

NCNitrocellulose membrane

membrane

NGE

Gold electrode with a nitrocellulose membrane

PCA Principal component analysis is a statistical procedure that uses an orthogonal transformation to convert a set of observations of possibly correlated variables into a set of values of linearly uncorrelated variables called principal components.

SVM A support vector machine (SVM) is a machine learning algorithm with a wide range of applications; it is especially good at building and forecasting binary classification models. Support vector regression (SVR) is a regression method based on an SVM.

SVR Support vector regression

Open Access This article is distributed under the terms of the Creative Commons Attribution 4.0 International License (http:// creativecommons.org/licenses/by/4.0/), which permits unrestricted use, distribution, and reproduction in any medium, provided you give appropriate credit to the original author(s) and the source, provide a link to the Creative Commons license, and indicate if changes were made.

\section{References}

1. Liu B, Luo Z, Zhang W, Tu Q, Jin X (2016) Carbon nanotube-based self-adhesive polymer electrodes for wireless long-term recording of electrocardiogram signals. J Biomater Sci Polym Ed 27(18): 1899-1908

2. Show PK, Sant S, Shukla A (2010) EIS studies on electroelectrodialysis cell for concentration of hydriodic acid. Int $\mathrm{J}$ Hydrog Energy 35(17):8868-8875
3. Zhang SS, Xu K, Jow TR (2006) EIS study on the formation of solid electrolyte interface in Li-ion battery. Electrochim Acta 51(8): $1636-1640$

4. Pietrantonio FD, Benetti M, Dinca Vet al (2014) Tailoring odorantbinding protein coatings characteristics for surface acoustic wave biosensor development. Appl Surf Sci 302(5):250-255

5. Shkrob A, Zhu Y, Marin TW et al (2016) Reduction of carbonate electrolytes and the formation of solid-electrolyte Interface (SEI) in lithium-ion batteries. 1. Spectroscopic observations of radical intermediates generated in one-Electron reduction of carbonates. J Phys Chem C 117(38):19255-19269

6. Osório WR, Freitas ES, Garcia A (2013) EIS and potentiodynamic polarization studies on immiscible monotectic Al-in alloys. Electrochim Acta 102(21):436-445

7. Badalyan A, Dierich M, Stiba K, Schwuchow V, Leimkühler S, Wollenberger U (2014) Lectrical wiring of the aldehyde oxidoreductase $\mathrm{PaOABC}$ with a polymer containing osmium redox centers: biosensors for benzaldehyde and GABA. Biosensors 4(4):403-421

8. Jiang J, Wang X, Chao R, Ren Y, Hu C, Xu Z, Liu GL (2014) Cellphone based portable bacteria pre-concentrating microfluidic sensor, and impedance sensing system. Sensors Actuators B Chem 193(3):653-659

9. Caviglia C, Zór K, Canepa S, Carminati M, Larsen LB, Raiteri R, Andresen TL, Heiskanen A, Emnéus J (2015) Interdependence of initial cell density, drug concentration and exposure time revealed by real-time impedance spectroscopic cytotoxicity assay. Analyst 140(10):3623-3629

10. Primiceri E, ChiriacòMS AED et al (2010) Real-time monitoring of copper ions-induced cytotoxicity by EIS cell chips. Biosens Bioelectron 25(12):2711-2716

11. Alfinito E, Millithaler JF, Pennetta C, Reggiani L (2010) A single protein based nanobiosensor for odorant recognition. Microelectron J 41(11):718-722

12. Lu Y, Zhang D, Zhang Q, Huang Y, Luo S, Yao Y, Li S, Liu Q (2016) Impedance spectroscopy analysis of human odorant binding proteins immobilized on nanopore arrays for biochemical detection. Biosens Bioelectron 79:251-257

13. Capone S, Francioso L, Siciliano P et al (2009) 138 electrical impedance spectroscopy of a pig odorant binding protein immobilized onto gold interdigited microelectrodes: an ab-initio study. AIP Conference Proceedings

14. Badalyan A, Neumann-Schaal M, Leimkühler S, Wollenberger U (2013) A biosensor for aromatic aldehydes comprising the mediator dependent PaoABC-aldehyde oxidoreductase. Electroanalysis 25(1):101-108

15. Robles CIG, Vázquez-Zapién GJ, Mata-Miranda MM et al (2017) Electrical bioimpedance spectroscopy as biosensor technique to identify cells lineages and cell differentiation process. Conf Proc IEEE Eng Med Biol Soc 3568-3571

16. Zou L, Wu C, Wang Q, Zhou J, Su K, Li H, Hu N, Wang P (2015) An improved sensitive assay for the detection of psp toxins with neuroblastoma cell-based impedance biosensor. Biosens Bioelectron 67:458-464

17. Hong WC (2009) Hybrid evolutionary algorithms in a SVR-based electric load forecasting model. Int J Electr Power Energy Syst 31(7-8):409-417

18. Fan G, Shan Q, Wang H et al (2014) Study on apparent kinetic prediction model of the smelting reduction based on the time-series. Math Probl Eng 2012(1024-123X):115-123

19. $\mathrm{Hu} \mathrm{Z}$, Bao Y, Xiong T (2013) Electricity load forecasting using support vector regression with memetic algorithms. Sci World J 2013(1):292575

20. Hu Z, Bao Y, Chiong R, Xiong T (2015) Mid-term interval load forecasting using multi-output support vector regression with a memetic algorithm for feature selection. Energy 84:419-431 
21. Nekkaa M, Boughaci D (2015) A memetic algorithm with support vector machine for feature selection and classification. Memet Comput 7(1):59-73

22. Suehiro J, Zhou G, Hara M (2003) Rapid communication: fabrication of a carbon nanotube-based gas sensor using dielectrophoresis and its application for ammonia detection by impedance spectroscopy. J Phys D Appl Phys 36(21):L109-L114

23. Shervedani RK, Mehrjardi AH, Zamiri N (2006) A novel method for glucose determination based on electrochemical impedance spectroscopy using glucose oxidase self-assembled biosensor. Bioelectrochemistry 69(2):201-208

24. Chen H, Heng CK, Puiu PD, Zhou XD, Lee AC, Lim TM, Tan SN (2005) Detection of Saccharomyces cerevisiae immobilized on self-assembled monolayer (SAM) of alkanethiolate using electrochemical impedance spectroscopy. Anal Chim Acta 554(1-2):52-59

25. Lu Y, Yao Y, Li S, Zhang Q, Liu Q (2017) Olfactory biosensor based on odorant-binding proteins of Bactrocera dorsalis with electrochemical impedance sensing for pest management. Sens Rev 37(4):396-403

26. Chmiola J, Largeot C, Taberna PL, Simon P, Gogotsi Y (2008) Desolvation of ions in subnanometer pores and its effect on capacitance and double-layer theory. Angew Chem 120(18):3440-3443

27. Shervedani RK, Mozaffari SA (2006) Impedimetric sensing of uranyl ion based on phosphate functionalized cysteamine self-assembled monolayers. Anal Chim Acta 562(2):223228

28. Creager SE, Wooster TT (1998) A new way of using ac voltammetry to study redox kinetics in electroactive monolayers. Anal Chem 70(20):4257-4263

29. Bogomolova A, Komarova E, Reber K, Gerasimov T, Yavuz O, Bhatt S, Aldissi M (2009) Challenges of electrochemical impedance spectroscopy in protein biosensing. Anal Chem 81(10):39443949

30. Farace G, Lillie G, Hianik T, Payne P, Vadgama P (2002) Reagentless biosensing using electrochemical impedance spectroscopy. Bioelectrochemistry 55:1-2):1-3

31. Mejri MB, Baccar H, Baldrich E, del Campo FJ, Helali S, Ktari T, Simonian A, Aouni M, Abdelghani A (2010) Impedance biosensing using phages for bacteria detection: generation of dual signals as the clue for in-chip assay confirmation. Biosens Bioelectron 26(4): 1261-1267

32. Lisdat F, SchãFer D (2008) The use of electrochemical impedance spectroscopy for biosensing. Anal Bioanal Chem 391(5):15551567

Publisher's note Springer Nature remains neutral with regard to jurisdictional claims in published maps and institutional affiliations.

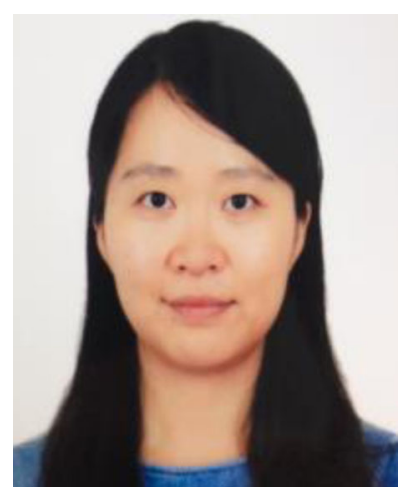

Ying $\mathrm{Xu}$, who received a doctoral degree from Zhejiang University in 2007, is now a master's degree tutor at Hangzhou Dianzi University and deputy director of the Department of Instrumental Science and Technology. Her main research directions include sensor microchip simulations, design and overall hardware platform construction, and later signal analysis work. Other research interests include organically combining the electrical performance of a specific sensor and the detected biological tissue unit. In 2009, she hosted the National Natural Science Foundation of China Youth Fund. In recent years, Ying Xu has published more than 20 papers and developed 3 patents.

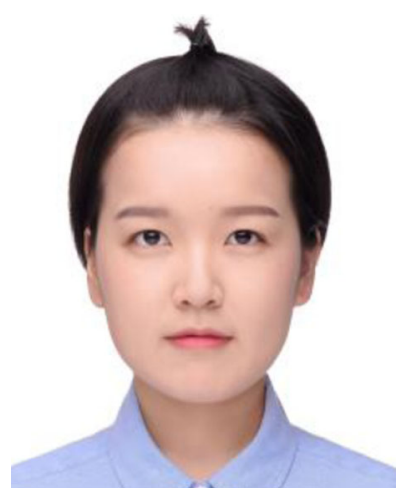

Chao $\mathbf{L i}$ is enrolled in the graduate division of the Department of Biomedical Engineering at Hangzhou Dianzi University. She has presided over two National Innovation and Entrepreneurship Training Programs of Undergraduates and participated in the development of two patents.

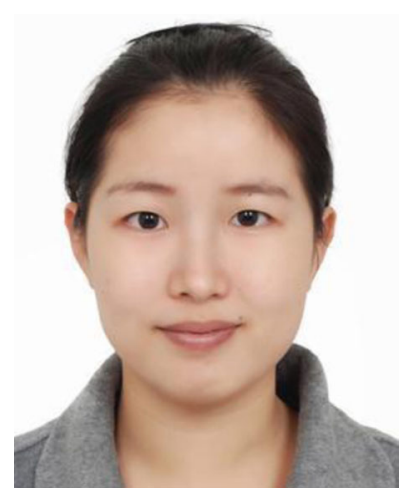

Mei Wanxin, a graduate student at Hangzhou Dianzi University from 2015 to 2018 , majored in Instrumental Science and Technology. In the past, she devoted her time to data analysis, especially of electrophysiological signals. In 2016, she published an article titled "Recognition and Classification of EEG Signals in Reading Mode Based on HilbertHuang Transformation." She has also studied the relationship among electrophysiological signals, cell impedance, and cell state to quantify cell activity. Recently, she has extensively researched subjects covering data analysis methods and their application to biomedical engineering, human engineering and cell engineering. 


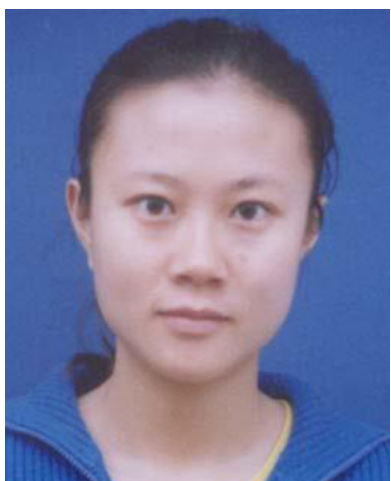

Miao Guo, who received a doctoral degree from Zhejiang University in 2006, is now a master's degree tutor at Hangzhou Dianzi University and deputy director of the Department of Biomedical Engineering. Her main research areas are biological monitoring and signal analysis. Miao Guo has published more than 10 papers in recent years.

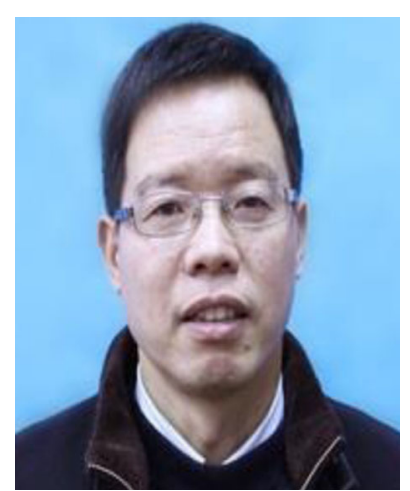

Yong Yang is a professor and doctoral supervisor at Hangzhou Dianzi University. He is mainly engaged in the research areas of medical instruments and image processing. He has hosted and participated in more than ten projects, such as the National Science and Technology Support Program, the National Natural Science Foundation, the National 863 Project subproject, and the Provincial Major Scientific and Technological Project. He has published more than ten articles in famous journals in China and abroad. He also participated in the publication of two monographs, one in a foreign language. He is a member of the Chinese Society of Biomedical Engineering and a deputy secretarygeneral of the Zhejiang Provincial Union of Innovative Medical Devices. 